\title{
INFLUÊNCIA DE MODELOS DE COEFICIENTE DE ARRASTE NO CÁLCULO DA RETENÇÃO GASOSA GLOBAL EM BIORREATOR COLUNA DE BOLHAS USANDO CFD
}

\author{
G. Y. RODRIGUEZ ${ }^{1}$, M. V. RAMIREZ ${ }^{1}$, R. BÉTTEGA ${ }^{1}$ e A. C. BADINO ${ }^{1}$ \\ ${ }^{1}$ Universidade Federal de São Carlos, Departamento de Engenharia Química \\ E-mail para contato: guilherme.ry@gmail.com
}

\begin{abstract}
RESUMO - Os biorreatores pneumáticos do tipo coluna de bolhas são equipamentos isentos de partes móveis e anteparos internos, onde a agitação e a mistura são realizadas exclusivamente pela injeção de ar (ou outro gás) na parte inferior. A retenção gasosa global é de extrema importância na avaliação do fenômeno de transferência de oxigênio em biorreatores, necessário em cultivos submersos aerados. No intuito de reduzir custos e otimizar o tempo de desenvolvimento de processos, as simulações em Fluidodinâmica Computacional (CFD - Computational Fluid Dynamics) vêm apresentando-se como uma poderosa ferramenta. O objetivo deste trabalho foi simular a hidrodinâmica de um biorreator pneumático do tipo coluna de bolhas operando com água e ar, utilizando CFD, e avaliar a retenção gasosa global sob diferentes vazões de alimentação ar. Foram empregados dois diferentes modelos de forças de arraste para que seus efeitos fossem avaliados nos resultados simulados de retenção gasosa global. O trabalho foi feito em um sistema de 5,5 L e os resultados foram comparados com os valores experimentais da literatura. As simulações em CFD conduzidas com o modelo que admite outros formatos geométricos de bolha apresentaram os melhores resultados de retenção gasosa global.
\end{abstract}

\section{INTRODUÇÃO}

Biorreatores ou reatores bioquímicos são equipamentos que fornecem um ambiente físico e químico adequado para a produção de um composto de valor agregado que é resultante da atividade catalítica de enzimas ou da rota metabólica de microrganismos como fungos e bactérias. Esses equipamentos operam a partir do ajuste e manutenção dos valores de $\mathrm{pH}$, temperatura, concentração de nutrientes e de oxigênio dissolvido a fim de que o produto de interesse seja gerado satisfatoriamente.

Os biorreatores pneumáticos do tipo coluna de bolhas são caracterizados pela ausência de partes móveis, utilizadas para promover a mistura do sistema reacional (Figura 1). A homogeneização é feita exclusivamente através da injeção de gás, em geral ar ou ar enriquecido com oxigênio, através de um aspersor localizado na base da coluna. Além disso, esses equipamentos não apresentam qualquer tipo de anteparo interno que induz a circulação dos fluidos. Essa modalidade de biorreator, comparativamente com o modelo convencional tipo tanque agitado e aerado, é capaz de reduzir o consumo de energia, aumentar a transferência de oxigênio, reduzir o cisalhamento celular e facilitar 
aspectos relacionados à implementação do sistema, como o projeto, construção e aumento de escala. Por isso, os biorreatores pneumáticos se mostram mais promissores atualmente em relação aos convencionais.

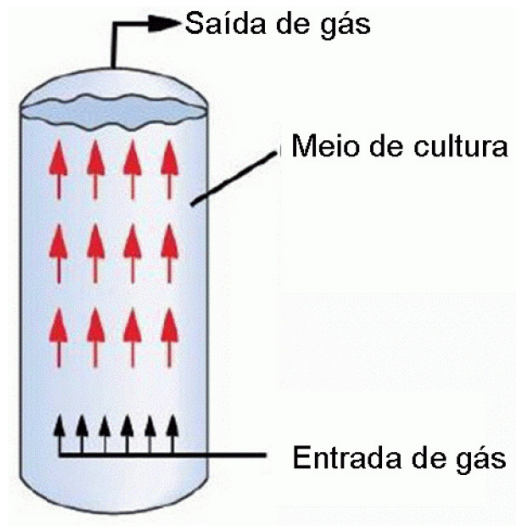

Figura 1 - Esquema simplificado de um biorreator do tipo coluna de bolhas (Cerri, 2005).

De acordo com Merchuk (2003), os biorreatores pneumáticos possuem aplicações no tratamento de efluentes e em processos bioquímicos diversos. São encontrados processos de desnitrificação, remoção de corantes em efluentes de indústrias têxteis, retirada de compostos fenólicos, oxidação de nitritos a nitratos, bio-oxidação de minerais, produção de enzimas celulolíticas por fermentação, produção de lipídeos a partir da cultura de microalgas, insumos para a produção de biocombustíveis, etc. O mesmo autor cita que o estudo da fluidodinâmica em biorreatores pneumáticos tem um grande apelo tecnológico, sendo um critério essencial na comparação entre sistemas.

\subsection{Variáveis e Parâmetros de Desempenho}

A vazão específica de ar $(\phi)$ é definida como sendo o quociente entre a vazão volumétrica de gás injetado $\left(\mathrm{Q}_{\mathrm{G}}\right)$ e o volume da fase líquida $\left(\mathrm{V}_{\mathrm{L}}\right)$, conforme mostra a Equação 1. A unidade mais usada para $\phi$ é $\min ^{-1}$, sendo que na terminologia tradicional denota-se por "vvm" (vazão de gás por volume de meio).

$$
\phi=\frac{Q_{G}}{V_{L}}
$$

A retenção gasosa global $(\bar{\alpha})$ corresponde à fração de ar presente na dispersão após a aeração. É o quociente entre o volume de gás $\left(V_{G}\right)$ e o volume da dispersão $\left(V_{L}+V_{G}\right)$, conforme apresentado na Equação 2.

$$
\bar{\alpha}=\frac{V_{G}}{V_{L}+V_{G}}
$$




\subsection{Objetivo}

A retenção gasosa global é um importante parâmetro de desempenho na avaliação de biorreatores pneumáticos e imprescindível em cultivos submersos e aerados, pois está relacionada à transferência de oxigênio. A Fluidodinâmica Computacional se apresenta como uma poderosa ferramenta numérica para reduzir custos e otimizar o tempo de desenvolvimento de processos.

Considerando os fatos expostos acima, o objetivo deste trabalho foi obter, usando CFD, valores de retenção gasosa global em um biorreator pneumático do tipo coluna de bolhas de 5,5 L capacidade útil operando com água e ar sob diferentes vazões específicas de alimentação do gás (1 a 6 vvm). Para cada vazão considerada, dois modelos de forças de arraste (Schiller e Naumann (1933); Grace et al. (1976)) foram testados. Os valores previstos pelos modelos foram comparados com resultados experimentais obtidos por Thomasi (2010).

\section{MATERIAIS E MÉTODOS}

\subsection{Aparato Experimental}

O aparato experimental utilizado (Figura 2.1) está apresentado no trabalho de Thomasi (2010). O biorreator coluna de bolhas possui uma base circular de $12,5 \mathrm{~cm}$ de diâmetro, altura total de $60 \mathrm{~cm}$ e altura de líquido de $45 \mathrm{~cm}$. O ar é injetado na base da coluna através de aspersor do tipo cruzeta, que possui 84 orifícios de $0,5 \mathrm{~mm}$ de diâmetro, espaçados igualmente em $5 \mathrm{~mm}$, conforme ilustra a Figura 2.2 .

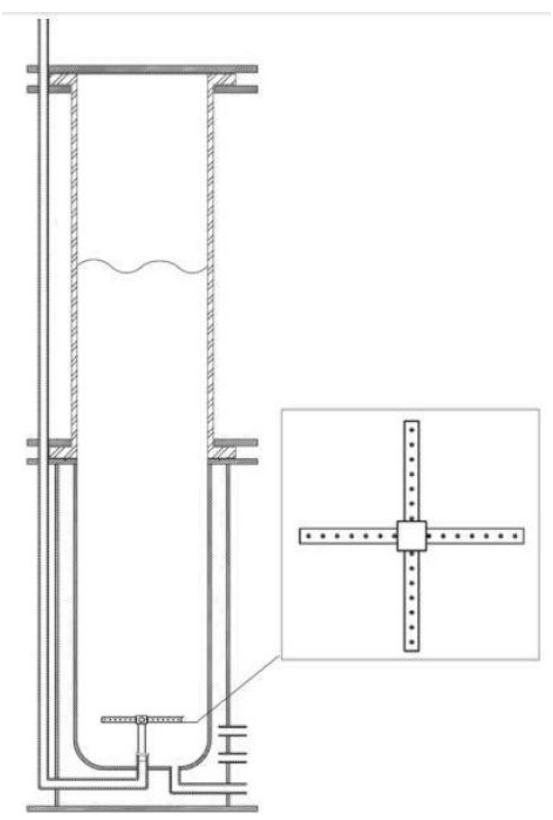

Figura 2.1 - Esquema do biorreator coluna de bolhas (Thomasi, 2010)

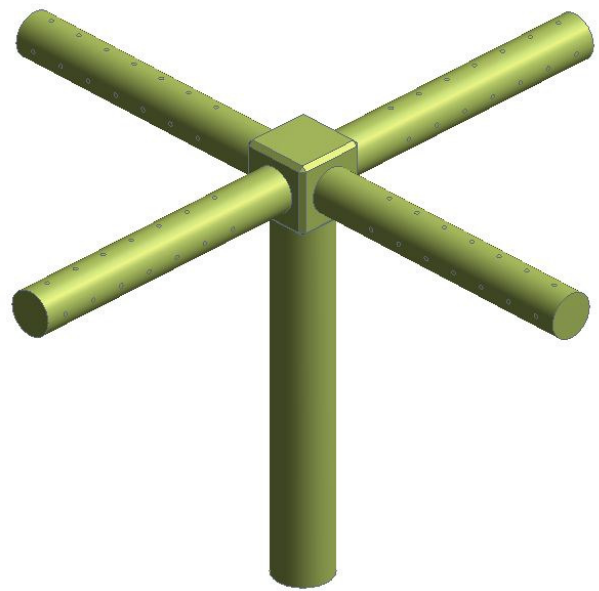

Figura 2.2 - Aspersor do tipo cruzeta 


\subsection{Software e Hardware}

As simulações em CFD foram conduzidas no software ANSYS FLUENT ${ }^{\circledR}$ 14.5. O hardware utilizado foi em servidor com placa-mãe Intel Server Board com 2 processadores Intel Xeon (12 núcleos por processador), 16 Gb de memória RAM e placa de vídeo QUADRO PNY $1 \mathrm{~Gb}$.

\subsection{Geometria Computacional e Malha}

A geometria computacional do biorreator coluna de bolhas foi criada no ANSYS Design Modeler $^{\circledR}$ (Figura 3.1). A malha foi gerada no Gambit v. 2.4 e possui aproximadamente 500.000 elementos (Figura 3.2), número obtido em teste de independência de malha. A malha foi composta por aproximadamente $85 \%$ de elementos tetraédricos (região do aspersor) e $15 \%$ hexaédricos.

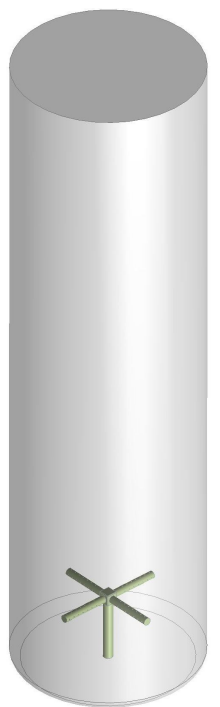

Figura 3.1 - Geometria computacional do biorreator coluna de bolhas

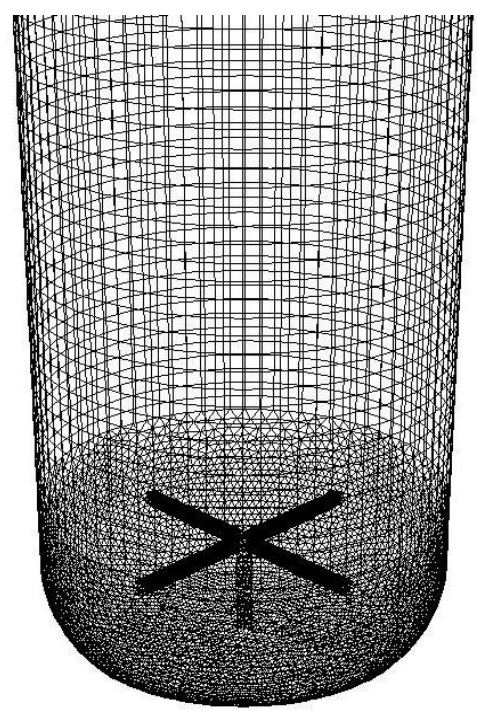

Figura 3.2 - Detalhe da malha na base da coluna

\subsection{Modelagem Matemática}

A Tabela 1 sumariza as condições mais relevantes usadas nas simulações.

Tabela 1 - Condições adotadas nas simulações

\begin{tabular}{|l|l|l|l|}
\hline Abordagem & Euler-Euler multifásico transiente & $\begin{array}{l}\text { Diâmetro médio de } \\
\text { bolha }\end{array}$ & $5 \mathrm{~mm}$ \\
\hline Fluidos & Água (primário) e ar (secundário) & Passo no tempo & $\begin{array}{l}1 \times 10^{-4} \mathrm{~s} \text { até } 0,5 \mathrm{~s} ; \\
1 \times 10^{-3} \mathrm{~s} \text { até } 5 \mathrm{~s}\end{array}$ \\
\hline Turbulência & k-E & Resíduo RMS & $1 \times 10^{-4}$ \\
\hline Discretização & Momentum (Second Order Upwind); Fração volumétrica (QUICK Scheme) \\
\hline
\end{tabular}

Os modelos de coeficiente de arraste propostos por Schiller e Naumann (1933) e Grace et al. (1976) estão representados pelas Equações 3 e 4, respectivamente. O primeiro modelo assume que as 
bolhas são esferas rígidas, enquanto que o segundo admite outras formas geométricas de acordo com as condições operacionais e propriedades do sistema.

$$
\begin{aligned}
& C_{D}=\max \left(\frac{24}{\operatorname{Re}_{b}}\left(1+0,15 \cdot R e_{b}^{0,687}\right) ; 0,44\right) \\
& C_{D}^{e l}=\frac{4}{3} \frac{g \cdot d_{b}}{v_{\infty}^{2}} \frac{\left|\rho_{L}-\rho_{G}\right|}{\rho_{L}} \quad \text { (para bolhas de formatos elípticos) }
\end{aligned}
$$

\section{RESULTADOS E DISCUSSÃO}

\subsection{Teste de Independência de Malha}

A fim de definir o número de elementos tridimensionais da malha, foram realizados testes de independência de malha conduzidos à 2 e 6 vvm (Figuras 4.1 e 4.2, respectivamente) com o modelo de Schiller e Naumann (1933). Nota-se que em 400.000 elementos os resultados de retenção gasosa global não diferiram significativamente daqueles obtidos usando 500.000 elementos. No entanto, optou-se por adotar a última malha, já que ela está mais refinada em regiões significativas, melhorando os resultados locais. Esse refinamento local na região do aspersor e na região da interface gás-líquido é necessário e foi recomendado por diversos autores, incluindo Blazej et al. (2004) e Hekmat et al. (2010). A avaliação do escoamento local em biorreatores pneumáticos será publicada em trabalhos subsequentes.

A metodologia utilizada para avaliar e monitorar a retenção global nos testes de independência de malha consistiu na aplicação do Teorema do Valor Médio (Equação 5), cuja rotina já está implementada na forma de uma função residente do ANSYS FLUENT ${ }^{\circledR} 14.5$.

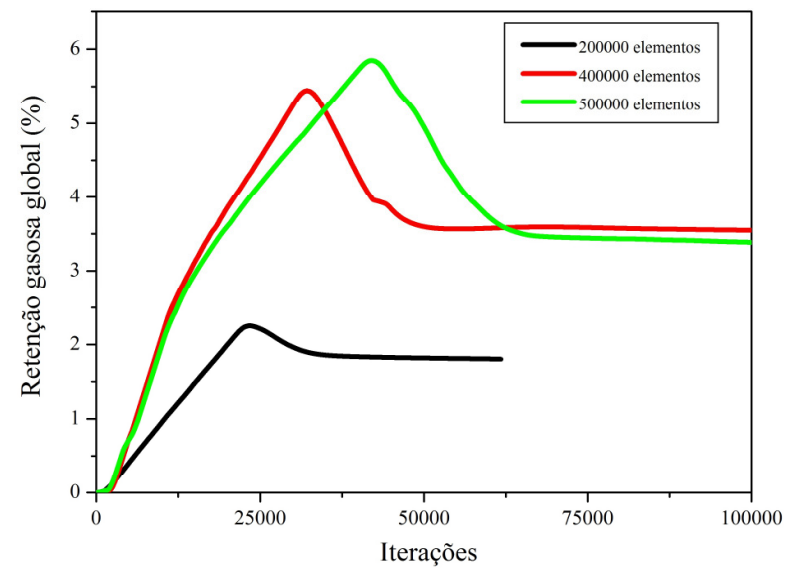

Figura 4.1 - Teste de independência de malha à $2 \mathrm{vvm}$

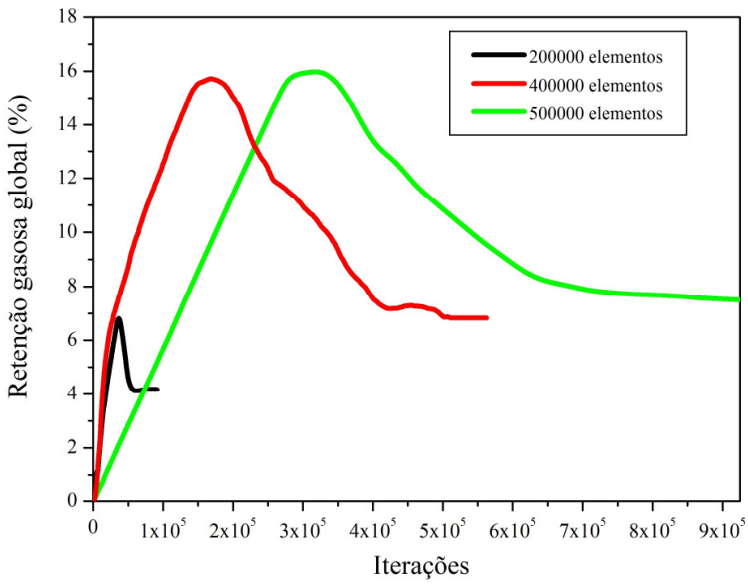

Figura 4.2 - Teste de independência de malha à 6 vvm 


$$
\bar{\alpha}_{G}=\frac{\iiint \alpha_{G} d V}{V}
$$

Deve ser ressaltado que a Equação 5 foi utilizada apenas como critério para o monitoramento do teste de independência de malha. Uma abordagem diferenciada foi empregada para comparar a retenção gasosa global com os resultados experimentais.

\subsection{Verificação da Influência dos Modelos de Coeficiente de Arraste}

Após definir o número de elementos da malha, foram conduzidos ensaios em CFD objetivando a avaliação de modelos para cálculo do coeficiente de arraste, propostos por Schiller e Naumann (1933) e Grace et al. (1976). A fim de que os resultados simulados possam ser comparados com aqueles obtidos por Thomasi (2010), foi necessário conduzir uma simulação transiente em CFD para cada vazão específica de 1 a 6 vvm.

A obtenção da retenção gasosa global via CFD foi similar ao método utilizado por Thomasi (2010), no qual esse parâmetro foi calculado através da manipulação da Equação 2, resultando em uma relação entre a altura inicial do líquido e a altura da dispersão após a aeração do sistema, conforme mostra a Equação 6.

$$
\bar{\alpha}_{G}=\frac{H_{D}-H_{L}}{H_{D}}
$$

Na Figura 5 são apresentados os resultados decorrentes das simulações usando os dois modelos de coeficiente de arraste propostos, onde cada ponto das curvas coloridas correspondeu a uma simulação transiente em CFD (aproximadamente 4 dias de simulação por ponto).

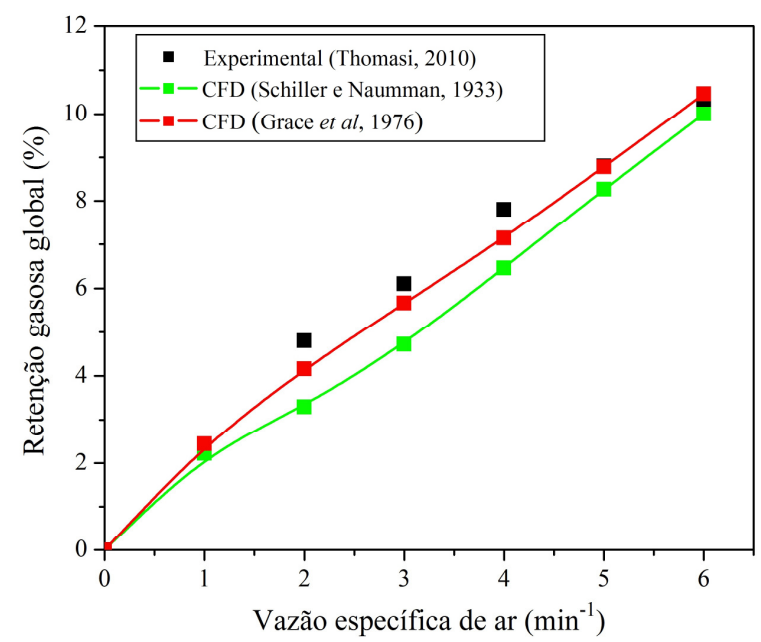

Figura 5 - Retenção gasosa global em função da vazão específica de ar

É possível verificar na Figura 5 que o modelo de Schiller e Naumann (1933) forneceu resultados de retenção gasosa global que subestimaram os valores experimentais, enquanto o modelo 


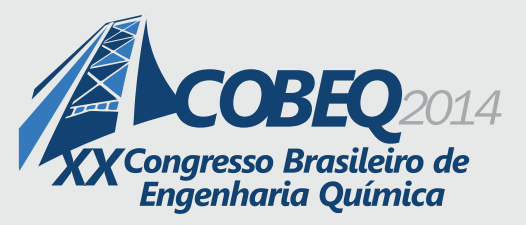

19 a 22 de outubro de 2014
Florianópolis/SC

de Grace et al. (1976) gerou resultados mais coerentes. O sistema água-ar é sabidamente coalescente, isto é, favorece o aparecimento de bolhas maiores que se formam a partir da união de bolhas pequenas. Nas Figuras 6.1 e 6.2 são apresentadas imagens do biorreator de coluna de bolhas usado neste trabalho operando com o sistema água-ar. Nesta figura é possível observar o aparecimento de bolhas amorfas (geometria indefinida), o que sugere a utilização de modelos que tentem compensar esta realidade.

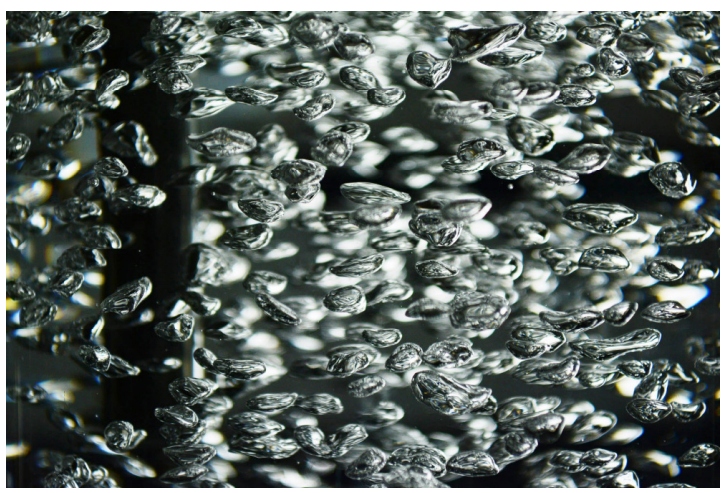

Figura 6.1 - Sistema água-ar a 1 vvm

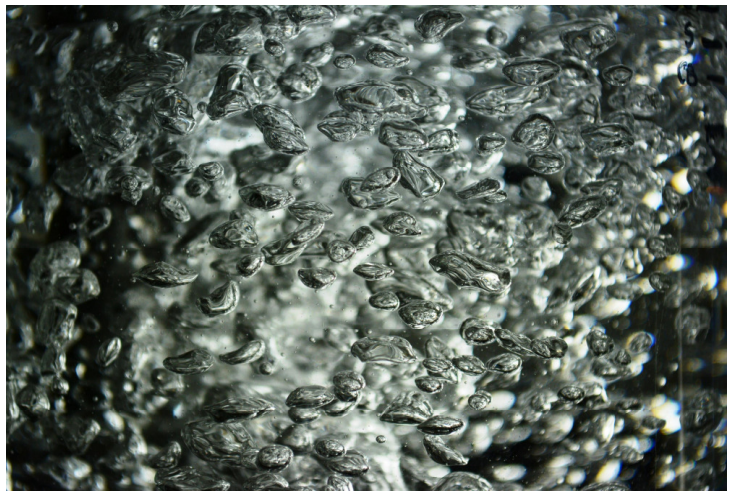

Figura 6.2 - Sistema água-ar a 6 vvm

Como o modelo proposto por Schiller e Naumann (1933) assume que as bolhas possuem apenas formatos esféricos, há uma dificuldade inerente na previsão do coeficiente de arraste, pois essas partículas no caso real são bem distorcidas e amorfas (geometricamente irregulares). O modelo de Grace et al. (1976) melhorou os resultados simulados de retenção gasosa global, pois é capaz de prever o coeficiente de arraste para bolhas no formato de esfera, elipse e touca. Essa melhora se deve ao fato de que essas possibilidades permitiram uma modelagem que tentou compensar os formatos geometricamente indefinidos que se observou no caso real.

\section{CONCLUSÃO}

O presente trabalho objetivou a verificação da influência de diferentes modelos, propostos por Schiller e Naumman (1933) e Grace et al. (1976), para o cálculo do coeficiente de arraste em um biorreator pneumático do tipo coluna de bolhas. Concluiu-se que o modelo de Grace et al. (1976) forneceu resultados de retenção gasosa global mais próximos da realidade, pois o mesmo considera que as bolhas possam assumir formatos geometricamente definidos, como esfera, elipse e touca.

\section{NOMENCLATURA}

L - Dimensão de comprimento; T - Dimensão de tempo; M - Dimensão de massa

$\mathrm{C}_{\mathrm{D}}^{\mathrm{elip}}$

$\mathrm{C}_{\mathrm{D}} \quad$ Coeficiente de arraste (-)

$\mathrm{H}_{\mathrm{D}} \quad$ Altura da dispersão gás-líquido (L)

$\mathrm{H}_{\mathrm{L}} \quad$ Altura inicial de líquido (L)

$\mathrm{Re}_{\mathrm{b}} \quad$ Número de Reynolds de bolha (-) 
Q $\quad$ Vazão volumétrica de alimentação de $\operatorname{ar}\left(\mathrm{L}^{3} \cdot \mathrm{T}^{-1}\right)$

$\mathrm{V}_{\infty} \quad$ Velocidade terminal da bolha $\left(\mathrm{L} . \mathrm{T}^{-1}\right)$

$\mathrm{V}_{\mathrm{G}} \quad$ Volume de ar presente no sistema após a aeração $\left(\mathrm{L}^{3}\right)$

$\mathrm{V}_{\mathrm{L}} \quad$ Volume de líquido avaliado antes da aeração do sistema $\left(\mathrm{L}^{3}\right)$

Letras gregas

$\bar{\alpha}_{\mathrm{G}} \quad$ Retenção gasosa global ou fração volumétrica global de ar (-)

$\phi \quad$ Vazão específica de ar $\left(\mathrm{T}^{-1}\right)$

$\rho_{\mathrm{G}} \quad$ Densidade do ar $\left(\mathrm{M}^{-L^{-3}}\right)$

$\rho_{\mathrm{L}} \quad$ Densidade do líquido (M.L ${ }^{-3}$ )

\section{REFERÊNCIAS}

BLAZEJ, M.; CARTLAND GLOVER, G. M.; GENERALIS, S. C.; MARKOS, J. Gas-liquid simulation of an airlift bubble column reactor. Chemical Engineering and Processing, v. 43, p. 137144, 2004.

CERRI, M. O. Avaliação de transferência de calor e massa de um biorreator airlift de circulação interna de bancada para a produção de ácido clavulânico. 116p. Dissertação (Mestrado em Engenharia Química), Universidade Federal de São Carlos, São Carlos, 2005.

GRACE, J. R.; WAIREGI, T.; NGUYEN, T. H. Shapes and velocities of single drops and bubbles moving freely through immiscible liquids. Transactions of the Institution of Chemical Engineers, v. 54, p. 167-173, 1976.

HEKMAT, A.; AMOOGHIN, A. E.; MORAVEJI, M. K. CFD simulation of gas-liquid flow behaviour in an air-lift reactor: determination of the optimum distance of the draft tube. Simulation Modeling Practice and Theory, v. 18, p. 927-945, 2010.

MERCHUK, J. C. Airlift bioreactors: review of recent advances. The Canadian Journal of Chemical Engineering, v. 81, p. 324-337, 2003.

SCHILLER, L. A.; NAUMANN, Z. A drag coefficient correlation. VDI Zeits, v. 77, p. 318, 1933.

THOMASI, S. S. Avaliação de parâmetros de desempenho de três modelos de biorreatores pneumáticos de bancada. 86f. Dissertação (Mestrado em Engenharia Química), Universidade Federal de São Carlos, São Carlos, 2010. 